\title{
Glutathione-Mediated Delivery and Release using Monolayer Protected Nanoparticle Carriers
}

\author{
Rui Hong, ${ }^{\dagger}$ Gang Han, ${ }^{\dagger}$ Joseph M. Fernández, ${ }^{\S}$ Byong-jin Kim, ${ }^{\ddagger}$ Neil S. Forbes ${ }^{\ddagger}$ \\ and Vincent M. Rotello*, $\dagger$ \\ Department of Chemistry ${ }^{\dagger}$, Department of Chemical Engineering ${ }^{*}$ and Department of \\ Biochemistry and Molecular Biology $y^{\S}$ University of Massachusetts, Amherst, \\ Massachusetts, 01003
}

\section{Supporting Information}

Synthesis of AuNP:

HSBDP and TTMA ligands were synthesized according to the reported procedures. ${ }^{1}$ Gold nanoparticles (Au-C8) were prepared by following the Brust-Schiffrin two-phase method, using 1-octanethiol as the capping ligand. ${ }^{2}$ To functionalize the nanoparticle surface, $30 \mathrm{mg}$ of Au-C8 nanoparticles were mixed with $4 \mathrm{mg}$ of HSBDP ligand and 120 $\mathrm{mg}$ of TTMA ligand in $20 \mathrm{~mL}$ of dichloromethane (DCM). The solution was stirred in the dark for about 6 hours during which time the nanoparticles precipitated out of solution. Methanol was added dropwisely to dissolve the nanoparticles and the solution was stirred for at least another 24 hours. Solvent was removed and the nanoparticles were washed with DCM at least five times until no fluorescence was seen in the solution (excited by a hand-hold UV lamp). The nanoparticles were then dried in vacuum and dissolved in MilliQ water. According to the HSBDP to TTMA ratio used in the place exchange reaction, and the number of ligands estimated from the size of the nanoparticle, an average number of 4-5 HSBDP molecules per AuNP was estimated. The AuNP solution was stored at $4{ }^{\circ} \mathrm{C}$ in the dark and was found to be stable for at least 6 months.

UV-VIS spectrum of AuNP was recorded and shown in Figure S1. A peak around 500 $\mathrm{nm}$ in the UV-VIS spectrum of AuNP is attributed to the absorbance of the Bodipy molecules, which are attached to the nanoparticle surface.

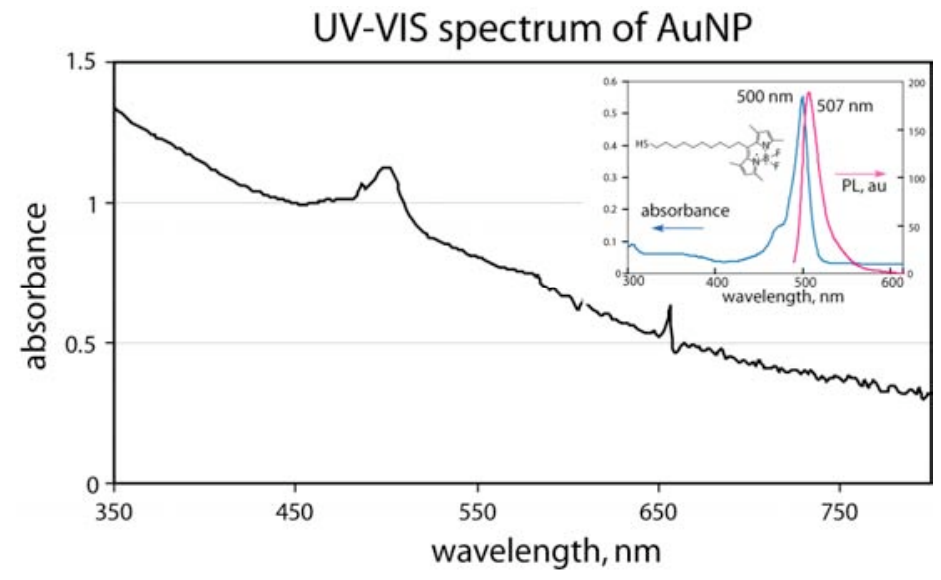

Figure S1. UV-VIS spectrum of AuNP in aqueous solution. Inset: UV-VIS and fluorescence spectra of HSBDP ligand. 
In vitro release:

AuNP stock solution $(10 \mu \mathrm{L}, 10 \mathrm{mg} / \mathrm{mL})$ was added to $500 \mu \mathrm{L}$ of freshly made GSH solution in a standard fluorescence cell. In control experiments, the control tripeptide, or MilliQ water was used instead of GSH. One milliliter of toluene was added carefully on the top of the aqueous solution. The cell was sealed and incubated in a $37{ }^{\circ} \mathrm{C}$ water bath. At predetermined time points, the fluorescence spectrum of the toluene phase was recorded, using a Shimadzu RF-5301 PC spectrofluorometer. The excitation wavelength was set at $485 \mathrm{~nm}$.

\section{Cell culture and imaging:}

Cells are grown in a cell culture flask in high glucose Dulbecco's Modified Eagle Medium (DMEM, 4.5g of glucose/L) containing $25 \mathrm{mM}$ HEPES buffer ( $\mathrm{pH} 7.4$ ) supplemented with $10 \%$ fetal bovine serum (FBS). Cultures are maintained at $37^{\circ} \mathrm{C}$ under humidified condition with $5 \% \mathrm{CO}_{2}$.

Florescent images were acquired on an inverted microscope (Olympus X71) with excitation 470/40 $\mathrm{nm}$ (center wavelength/width) and emission at 525/50 nm.

Inoculation cell density: 1000 cells/well (96-well plate).

Mouse fibroblast cells incubated with AuNP and imaged after 96 hours:

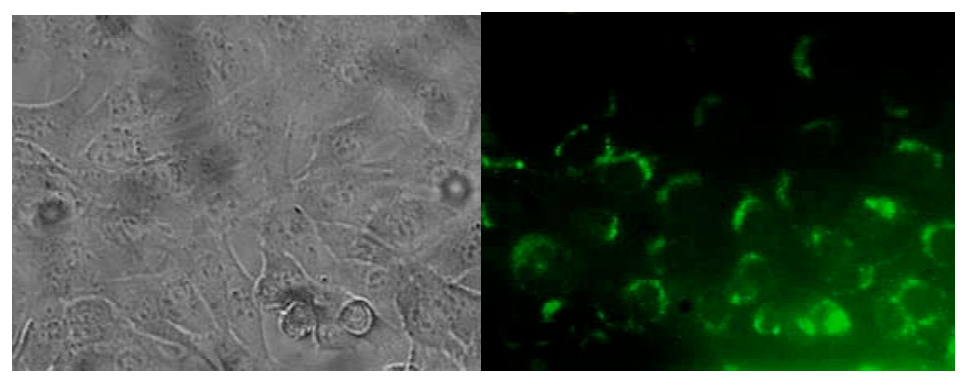

Figure S2. Representative fluorescence images of mouse fibroblast cells incubated with AuNP (for 8 hours) and imaged after 96 hours.

1) Shepherd, J. L.; Kell, A.; Chung, E.; Sinclar, C. W.; Workentin, M. S.; Bizzotto, D. J. Am. Chem. Soc. 2004, 126, 8329-8335. Hong, R.; Fischer, N. O.; Emrick, T.; Rotello, V. M. Chem. Mater. 2005, 17, 46174621.

2) Brust, M.; Walker, M.; Bethell, D.; Schiffrin, D. J.; Whyman, R. J. Chem. Soc., Chem. Commun. 1994, 7, 801. 